\title{
Falta de acesso e trajetória de utilização de serviços de saúde por idosos brasileiros
}

\author{
Lack of access and the trajectory of healthcare use by elderly Brazilians
}

Ana Paula Santana Coelho Almeida (https://orcid.org/0000-0001-5808-5818) ${ }^{1}$

Bruno Pereira Nunes (https://orcid.org/0000-0002-4496-4122) ${ }^{2}$

Suele Manjourany Silva Duro (https://orcid.org/0000-0001-5730-0811) ${ }^{2}$

Rita de Cássia Duarte Lima (https://orcid.org/0000-0002-5931-398X) ${ }^{3}$

Luiz Augusto Facchini (https://orcid.org/0000-0002-5746-5170) ${ }^{4}$
${ }^{1}$ Departamento de Medicina Social, Universidade Federal do Espírito Santo (UFES). Av. Marechal Campos 1468, Bairro Bonfim. 29047105 Vitória ES Brasil apscoelho@gmail.com ${ }^{2}$ Departamento de

Enfermagem em Saúde Coletiva, Universidade Federal de Pelotas (UFPEL)

Pelotas RS Brasil.

${ }^{3}$ Programa de Pós-

Graduação em Saúde Coletiva, UFES. Vitória ES

Brasil.

${ }^{4}$ Programa de Pós-

Graduação em

Epidemiologia,

Departamento de Medicina

Social, Faculdade de

Medicina, UFPEL. Pelotas

RS Brasil.

\begin{abstract}
Populational ageing is a current phenomenon and calls for the reconfiguration of health services and expansion of access for the elderly. This is a cross-sectional study with 6,624 elderly Brazilians over 60 that set out to evaluate access to healthcare by measuring lack of access and by describing the trajectory until recourse to hospitalization, emergency care and medical visits. The variables were analyzed according to the nature of funding to access the service (SUS, private insurance and cash-payment). The prevalence of lack of access was 2.5\% (CI95\% 1.6;4.0) for hospitalizations, $2.1 \%$ (CI95\% 1.4; 3.1) for emergency rooms, and $0.6 \%$ (CI95\% 0.3;0.9) for medical visits. SUS accounted for most of the care provided. Positive aspects of SUS were the higher number of medical visits in the city of residence and less money spent on transport. The private system stands out for the low frequency of long waiting times and higher frequency of referrals to return visits after hospitalization. The findings highlight the importance of SUS in Brazil in promoting equality and universality despite the existing deficiencies. In both public and private systems, greater articulation among the healthcare levels is required for integral healthcare to elderly individuals.

Key words Elderly, Access to Health Services, Health Systems, Unified Health System, Health Insurance
\end{abstract}

Resumo O envelhecimento populacional é um fenômeno atual e demanda reconfiguração e ampliação do acesso aos serviços de saúde para a população idosa. Estudo transversal, com amostra representativa de 6.624 idosos brasileiros, que teve como objetivo avaliar o acesso aos serviços de saúde por meio da falta de acesso e da descrição da trajetória do uso em internação, pronto-socorro e consulta médica. As variáveis foram analisadas segundo natureza do financiamento (SUS, convênio e desembolso direto). A prevalência de falta de acesso foi 2,5\% (IC95\% 1,6; 4,0) na internação, 2,1\% em pronto-socorro (IC95\% 1,4; 3,1), e 0,6\% (IC95\% 0,3; 0,9) no atendimento médico. O SUS foi responsável pela maioria dos atendimentos. Aspectos positivos do SUS foram maior número de atendimentos médicos realizados na cidade de residência e menor frequência de dinheiro gasto no deslocamento. O privado se destacou pela baixa frequência de tempo de espera longo e maior frequência de encaminhamentos para consulta de revisão após internação. Os achados reforçam a importância do SUS na promoção da equidade e universalidade apesar das deficiências existentes. Tanto no sistema público quanto no privado fazse necessário articulação dos níveis de atenção na perspectiva da integralidade no cuidado ao idoso. Palavras-chave Idoso, Acesso aos Serviços de Saúde, Sistemas de Saúde, Sistema Único de Saúde, Seguro Saúde 


\section{Introdução}

A mudança no perfil e na dinâmica demográfica mundial tem como manifestação a tendência de envelhecimento da população. No Brasil, esse fenômeno se iniciou mais recentemente e de forma mais rápida, quando comparado a países mais desenvolvidos, trazendo consigo grande impacto social, exigindo mudanças nas políticas públicas ${ }^{1}$. Na saúde, esse cenário demanda a reconfiguração dos serviços, com readequação da oferta e qualificação de recursos humanos, garantindo acesso para atendimento das necessidades de saúde dessa população.

Os idosos são grandes usuários do sistema de saúde. No Brasil, a prevalência de utilização de consulta médica nos 12 meses anteriores à entrevista, foi de 79,3\% de acordo com a Pesquisa Nacional de Saúde (PNS) de 2013². Importantes avanços têm sido apontados no país em relação à redução das desigualdades sociais no uso de serviços de saúde por idosos. Inquérito realizado em 2009 não identificou diferença estatística na associação entre atendimento médico e classificação econômica, entre idosos com diabetes ${ }^{3}$.

De maneira geral, o acesso tem sido conceituado como o grau de facilidade com que as pessoas obtêm cuidados de saúde, ou como a oportunidade de ingressar no sistema de saúde ${ }^{4}$. A utilização de serviço de saúde é caracterizada pela garantia do atendimento, ou seja, se refere à parcela da população que procurou o serviço de saúde e obteve êxito ${ }^{4}$. Há crescente disponibilidade de trabalhos sobre utilização de serviços de saúde no Brasil ${ }^{5}$, entretanto são escassos estudos observacionais que abordem outras dimensões do acesso. Devido à dificuldade de se obter medidas de acesso em seu domínio mais amplo, a maior parte dos estudos tem adotado a utilização de serviços de saúde como proxy do acesso.

Embora ainda exista considerável debate sobre o conceito de acesso à saúde, parte dos autores concordam que acesso não equivale à utilização do serviço de saúde ${ }^{4,6,7}$. Essa medida não permite conhecer a respeito daqueles indivíduos que não conseguiram atendimento. Propõe-se, nessa perspectiva, a mensuração da falta de acesso compreendida como a proporção de pessoas que necessitaram de cuidados de saúde, procuraram o serviço, mas não conseguiram o atendimento.

O sistema de saúde brasileiro possui um formato peculiar em relação a outros países no que se refere ao financiamento, prestação de serviços, arranjos e natureza dos prestadores ${ }^{8,9}$. A proteção social da saúde é garantida por meio do Siste- ma Único de Saúde (SUS) que se constitui num sistema público, de caráter nacional e universal. No entanto, o SUS coexiste com um forte sistema privado, constituído por planos e seguros de saúde e ainda há contratação de serviços via desembolso direto ${ }^{8}$.

Conhecer a trajetória do usuário até a efetivação do uso tanto no público quanto no privado é fundamental na avaliação do desempenho do sistema de saúde. Muitos estudos com abordagem qualitativa se empenharam em abordar a temática ${ }^{10}$, ao passo que estudos observacionais com detalhamento da trajetória da utilização de serviços de saúde entre idosos são raros. É escasso também esse tipo de estudo comparando prestações de serviços públicos e privados.

Buscando contribuir para o conhecimento sobre o desempenho do sistema de saúde, o presente trabalho tem como objetivo avaliar o acesso aos serviços de saúde em uma amostra de idosos brasileiros por meio da mensuração da falta de acesso e da descrição da trajetória até a efetivação do uso, segundo a natureza pública (SUS) ou privada (convênio e desembolso direto) do financiamento.

\section{Método}

Estudo transversal de base nacional, realizado em 2009, que avaliou acesso aos serviços de saúde no Brasil, com ênfase no desempenho e na qualidade dos cuidados de saúde - AQUARES. Para o presente estudo utilizou-se amostra composta por idosos com 60 anos ou mais de idade, residentes em 100 municípios brasileiros de pequeno, médio e grande porte, de 23 estados das cinco regiões geopolíticas do Brasil. Foram incluídos no estudo idosos residentes na zona urbana, moradores em domicílios particulares e coletivos.

O tamanho da amostra foi estimado com base nas prevalências do desfecho encontradas no estudo de Green et al. ${ }^{11}$. Considerando uma prevalência de falta de acesso de $0,7 \%$, assumindo um erro de 0,2 pp e um efeito de delineamento de 1,4 , incluindo $15 \%$ para perdas e recusas, seria necessário estudar 6.000 idosos.

Foi realizado um processo de amostragem por múltiplos níveis, com igual probabilidade de seleção para os indivíduos. O primeiro nível foi representado pelo porte do município (menor de 10.000 , de 10.000 a 19.999 , de 20.000 a 99.999 , de 100.000 a 1,1 milhão, maior ou igual a $1,1 \mathrm{mi}-$ lhão), o segundo pelo município, o terceiro por setores censitários urbanos, o quarto por domicí- 
lios e o quinto, e último nível, por indivíduos elegíveis residentes nos domicílios. Dentro de cada grupo de acordo com o porte, os municípios foram sorteados aleatoriamente (pelo acaso não foi sorteado nenhum município do Amapá, Amazonas, Distrito Federal e Roraima). Em cada município os setores censitários urbanos também foram definidos através de sorteio aleatório. Em cada setor foram localizados 30 domicílios, com início aleatório e "pulo" sistemático de 10 domicílios. Em cada domicílio foram incluídos todos os indivíduos elegíveis, ainda que se ultrapassasse a cota de indivíduos definida no setor censitário.

A coleta de dados foi realizada em 2009 por 55 entrevistadores selecionados e treinados que utilizaram um dispositivo de mão (PDA - Personal Digital Assistant). Os dados foram armazenados em computadores portáteis e transferidos para a sede de estudo via Internet. $\mathrm{O}$ instrumento utilizado para a coleta de dados foi padronizado e pré-testado, e era composto por questões socioeconômicas, demográficas, bem como por questões relativas à utilização de serviços de saúde tanto no público quanto no privado.

Os entrevistados foram questionados se receberam algum cuidado de saúde recentemente (internação e cuidado de emergência nos últimos 12 meses; consulta médica nos últimos três meses), a fim de investigar utilização. A falta de acesso foi caracterizada quando um indivíduo referiu ter precisado de atendimento de saúde, ter buscado o serviço e não ter conseguido o atendimento. Foi mensurada através da seguinte sequência de questionamentos: "Desde < três meses atrás > o(a) $\mathrm{Sr}(\mathrm{a})$. foi atendido por algum médico?", Se não: "Apesar de não ter sido atendido por médico, o(a) $\mathrm{Sr}(\mathrm{a})$. precisou deste atendimento?", Se precisou: "O(A) $\mathrm{Sr}(\mathrm{a})$ buscou atendimento médico desde < três meses atrás $>$ ?", Se buscou atendimento médico: "Por qual motivo não foi atendido?”. As questões para investigar internação hospitalar, atendimento em pronto-socorro seguiram o mesmo padrão, entretanto se referiam a um período de 12 meses.

Questões coletadas a fim de investigar a trajetória até a efetivação do uso foram: se o indivíduo foi encaminhado (encaminhado ou conta própria) e qual serviço encaminhou para aquele serviço de saúde (Unidade de Saúde, Pronto-atendimento do convênio, ambulatório do hospital, consultório por convênio, consultório particular, outros); tempo de espera para conseguir o atendimento (mesmo dia, 1 a 5 dias, 6 a 15 dias, 16 a 30 dias, 31 a 60 dias, mais de 60 dias); se foi atendido no município onde mora ( $\mathrm{sim} /$ não); tempo de transporte até outro município (15 minutos ou menos, 16 a 30 minutos, 31 a 60 minutos, 61 a 120 minutos, mais de $120 \mathrm{minu}$ tos); motivos pelos quais teve que ir até outro município (não tem médico, não atendem este tipo, não gosta do atendimento, não resolvem, outro motivo); formas de transporte até o local de atendimento (caminhando, Ônibus/lotação/ Van/Kombi, Ambulância/SAMU, carro particular, taxi, outra maneira); gastos com transporte ( $\operatorname{sim} /$ não); se recebeu encaminhamento ( $\operatorname{sim} /$ não); e se conseguiu atendimento no local para onde foi encaminhado ( $\operatorname{sim} /$ não). Todas as perguntas se referiam ao último atendimento médico, última internação ou último atendimento em pronto-socorro.

Essas variáveis foram analisadas de acordo com a natureza do financiamento para acessar o serviço. O financiamento foi categorizado em convênio, quando os serviços prestados eram contratados por convênios, planos e seguros de saúde; particular, quando obtidos mediante desembolso direto e SUS, quando prestados pelo sistema público.

Variáveis descritivas individuais foram sexo (masculino/feminino); raça/cor auto-declarada (branca, preta, parda, indígena e amarela); idade em anos (60 a 64, 65 a 69, 70 a 79, 80 ou mais); educação em anos (nenhum, de um a quatro, de cinco a oito, de nove a onze, doze a mais); classificação econômica de acordo com a Associação Brasileira de Empresas de Pesquisa (ABEP) (A/B, $\mathrm{C}$ e $\mathrm{D} / \mathrm{E})$; renda per capita média em salários mínimos; região geopolítica do país (Centro-Oeste, Nordeste, Norte, Sudeste e Sul); e população do município (até 20.000; 20.001 a 100.000; 100.001 a 500.000; mais de 500.000 habitantes).

A análise foi realizada por meio da prevalência das variáveis e seus respectivos intervalos de confiança. Utilizou-se o pacote estatístico Stata $13.1^{\circledast}$ e o módulo svy foi empregado para o cálculo dos erros padrão, considerando o delineamento complexo do processo de amostragem da pesquisa.

O estudo foi aprovado pelo Comitê de Ética da Faculdade de Medicina da Universidade Federal de Pelotas. Todos entrevistados assinaram um termo de consentimento livre e esclarecido.

\section{Resultados}

Foram identificados 7.015 idosos. Perdas e recusas totalizaram 5,6\%, resultando em 6.624 indivíduos incluídos no estudo, dos quais 59\% (IC95\% 
58,0-59,9) eram mulheres, 52,9\% (IC95\% 50,954,8 ) declararam-se pardos e 40,3\% (IC95\% $38,4-42,3)$, brancos. A média de idade foi 70,9 anos (DP $=8,0$ anos) e 34,1\% (IC95\% 32,9$35,4)$ possuíam entre 70 e 79 anos de idade. A proporção de idosos que não completaram ao menos um ano de estudo foi de 37,8\% (IC95\% $35,7-40,0)$. A maioria dos indivíduos pertencia a famílias da classe C $(41,8 \%$ - IC95\% 39,9-43,6), e a média de renda per capita da amostra foi de 1,3 salários mínimos $(\mathrm{DP}=1,6)$. A maior parte dos respondentes residia na região sudeste do país (35,3\% - IC95\% 31,6-39,1), em municípios com mais de 500 mil habitantes (32,3\% - IC95\% 28,8-36,1) (Tabela 1).

\section{Atendimento médico}

Nos três meses anteriores a entrevista, $46,0 \%$ (IC95\% 44,4-47,6) dos idosos receberam atendimento médico e 1,5\% (IC95\% 1,1-2,0), referiu a necessidade de atendimento, apesar de não ter sido atendido. Desses, 32,1\% (IC95\% 19,8- 47,4) buscaram o atendimento, resultando em $0,6 \%$ (IC95\% 0,3- 0,9) de falta de acesso (Tabela 2).

Dentre aqueles que consultaram, 27,8\% (IC95\% 25,4-30,4) o fizeram via convênio, 10,4\% (IC95\% 9,3- 11,6) por desembolso direto e 61,8\% (IC95\% 59,0-64,5) via SUS. A maioria dos idosos buscou o serviço por conta própria nos três tipos de financiamento $(88,0 \%$ - IC95\% 86,589,3 ). Dos que utilizaram o SUS, $13,8 \%$ (IC95\% 12,0-15,9) chegaram ao serviço por encaminhamento, contra 10,3\% (IC95\% 8,3-12,6) dos que utilizaram convênio e 6,4\% (IC95\% 4,2-9,6) dos que utilizaram via particular. A Unidade Básica de Saúde foi a maior responsável pelos encaminhamentos do SUS $(77,0 \%$ - IC95\% 71,2-81,8) (Tabela 3).

Cerca de metade dos idosos conseguiu atendimento no mesmo dia, independentemente do tipo de financiamento. Espera de até cinco dias foi observada para 25,9\% (IC95\% 22,6-29,4) dos usuários do convênio, 27,9\% (IC95\% 23,1-33,4) dos que utilizaram via particular e 15\% (IC95\% 13,1-17,0) dos idosos que foram atendidos pelo SUS. Os usuários do SUS foram os que tiveram maior frequência de tempo de espera superior a 60 dias (6,9\% - IC95\% 5,6-8,5).

A maioria dos que consultaram pelo SUS foi caminhando até o local de atendimento $(57,9 \%$ - IC95\% 55,2-60,7), e somente 13,6\% (IC95\% 11,0-16,8) dos usuários do convênio e 13,1\% (IC95\% 9,8-17,4) do particular utilizaram essa forma de deslocamento. Carro particular foi o
Tabela 1. Descrição de características demográficas e socioeconômicas da amostra. Brasil, 2009.

\begin{tabular}{|c|c|c|c|}
\hline Variáveis & $\mathbf{n}$ & $\%$ & IC95\% \\
\hline \multicolumn{4}{|l|}{$\operatorname{Sexo}(n=6.616)$} \\
\hline Masculino & 2714 & 41,0 & $40,1-42,0$ \\
\hline Feminino & 3902 & 59,0 & $58,0-59,9$ \\
\hline \multicolumn{4}{|l|}{ Cor da pele $(n=6.456)$} \\
\hline Branca & 2604 & 40,3 & $38,4-42,3$ \\
\hline Preta & 376 & 5,8 & $5,1-6,6$ \\
\hline Parda & 3412 & 52,9 & $50,9-54,8$ \\
\hline Indígena/amarela & 64 & 1,0 & $0,8-1,3$ \\
\hline \multicolumn{4}{|c|}{$\begin{array}{l}\text { Idade em anos completos } \\
(\mathrm{n}=6.601)\end{array}$} \\
\hline 60 a 64 & 1661 & 25,2 & $24,0-26,3$ \\
\hline 65 a 69 & 1647 & 25,0 & $23,9-26,1$ \\
\hline 70 a 79 & 2251 & 34,1 & $32,9-35,4$ \\
\hline 80 ou mais & 1042 & 15,8 & $14,9-16,8$ \\
\hline \multicolumn{4}{|l|}{$\begin{array}{l}\text { Estado civil atual } \\
(\mathrm{n}=6.613)\end{array}$} \\
\hline Solteiro & 685 & 10,4 & $9,5-11,3$ \\
\hline Casado & 3384 & 51,2 & $49,6-52,8$ \\
\hline Separado/divorciado & 483 & 7,3 & $6,6-8,0$ \\
\hline Viúvo & 2061 & 31,2 & $29,9-32,4$ \\
\hline \multicolumn{4}{|l|}{$\begin{array}{l}\text { Escolaridade em anos } \\
\text { completos }(\mathrm{n}=6.538)\end{array}$} \\
\hline Nenhum & 2470 & 37,8 & $35,7-40,0$ \\
\hline Um a quatro & 2408 & 36,8 & $35,2-38,5$ \\
\hline Cinco a oito & 851 & 13,0 & $12,0-14,1$ \\
\hline Nove ou mais & 809 & 12,4 & $11,0-13,9$ \\
\hline \multicolumn{4}{|c|}{$\begin{array}{l}\text { Classificação econômica } \\
\text { (Abep) }(\mathrm{n}=6.346)\end{array}$} \\
\hline $\mathrm{A} / \mathrm{B}$ & 1079 & 17,0 & $15,3-18,1$ \\
\hline $\mathrm{C}$ & 2650 & 41,8 & $39,9-43,6$ \\
\hline $\mathrm{D} / \mathrm{E}$ & 2617 & 41,2 & $38,9-43,6$ \\
\hline \multicolumn{4}{|l|}{ Região (n=6.624) } \\
\hline Centro-Oeste & 707 & 10,7 & $8,5-13,4$ \\
\hline Nordeste & 1514 & 22,9 & $19,7-26,4$ \\
\hline Norte & 558 & 8,4 & $6,5-10,9$ \\
\hline Sudeste & 2338 & 35,3 & $31,6-39,1$ \\
\hline Sul & 1507 & 22,8 & $19,6-26,2$ \\
\hline \multicolumn{4}{|l|}{$\begin{array}{l}\text { Porte populacional } \\
(\mathrm{n}=6.624)\end{array}$} \\
\hline até 20.000 & 719 & 10,9 & $8,6-13,6$ \\
\hline 20.001 a 100.000 & 1754 & 26,5 & $23,1-30,1$ \\
\hline 100.001 a 500.000 & 2009 & 30,3 & $26,9-34,0$ \\
\hline Mais de 500.000 & 2142 & 32,3 & $28,8-36,1$ \\
\hline
\end{tabular}

meio de transporte mais utilizado pelos idosos que consultaram pelo convênio (53,8\% - IC95\% $49,8-57,7)$ e particular $(53,7 \%$ - IC $95 \% 47,8$ - 
Tabela 2. Falta de acesso no atendimento médico, na internação hospitalar e no atendimento em pronto-socorro. Brasil, 2009.

\begin{tabular}{|c|c|c|c|}
\hline & $\mathbf{n}$ & $\%$ & 95\%IC \\
\hline Atendimento médico $(\mathrm{n}=6.590)$ & 3.029 & 46,0 & $44,4-47,6$ \\
\hline Apesar de não ter sido atendido, precisou de atendimento $(\mathrm{n}=3.561)$ & 53 & 1,5 & $1,1-2,0$ \\
\hline Buscou atendimento $(n=53)$ & 17 & 32,1 & $19,8-47,4$ \\
\hline Falta de acesso na Consulta Médica $(\mathrm{n}=3.046)$ & 17 & 0,6 & $0,3-0,9$ \\
\hline Internação hospitalar $(\mathrm{n}=6.592)$ & 732 & 11,1 & $10,3-12,0$ \\
\hline Apesar de não ter internado, houve indicação médica para internar $(\mathrm{n}=5.860)$ & 19 & 0,32 & $0,2-0,5$ \\
\hline Falta de acesso na internação $(\mathrm{n}=751)$ & 19 & 2,5 & $1,6-4,0$ \\
\hline Pronto-Socorro $(\mathrm{n}=6.591)$ & 1.084 & 16,4 & $15,3-17,6$ \\
\hline Apesar de não ter sido atendido, precisou de atendimento $(\mathrm{n}=5.507)$ & 23 & 0,4 & $0,3-0,6$ \\
\hline Falta de acesso no Pronto-Socorro $(\mathrm{n}=1.107)$ & 23 & 2,1 & $1,4-3,1$ \\
\hline
\end{tabular}

59,5). Apenas 16,8\% (IC95\% 15,1-18,7) dos atendidos pelo SUS citaram essa forma de transporte.

Dos respondentes que consultaram pelo SUS, 9,5\% (IC95\% 8,1-11,2) gastaram algum dinheiro com transporte, contra 20,8\% (IC95\% 17,8-24,1) dos usuários do convênio e 28,5\% (IC95\% 23,3$34,4)$ daqueles atendidos via particular.

Os idosos atendidos pelo convênio (15,2\% IC95\% 12,1-18,9) e particular (24,6\% - IC95\% 19,6-30,4) apresentaram maior frequência de atendimento fora do município de residência, quando comparados aos que utilizaram o SUS (5,7\% - IC95\% 4,6-7,0). O motivo mais citado para consultar em outra cidade foi não existir o tipo de atendimento na cidade (34,1\% - IC95\% $27,8-41,0)$. Dentre os pacientes que utilizaram o convênio e particular, 20,3\% (IC95\% 13,9-28,6) e 26\% (IC95\% 16,9-37,6) respectivamente, referiram ter consultado em outro município por não gostar do atendimento em sua cidade, enquanto 9,7\% (IC95\% 5,0-18,0) dos atendidos pelo SUS citaram essa justificativa.

Dentre os idosos que foram atendidos fora do município de residência, cerca de um terço dos respondentes referiram um tempo de deslocamento de 31 a 60 minutos até o município de atendimento e 14,3\% (IC95\% 10,4-19,3), um tempo superior a 120 minutos, sem diferença entre as formas de financiamento.

Os usuários de convênio foram os que menos receberam encaminhamento para atendimento em outro local após a consulta (4,8\% - IC95\% 3,5-6,6), em contraposição aos usuários do SUS (8,2\% - IC95\% 7,0-9,7), em sua maioria encaminhados para uma unidade básica de saúde (30,9\% - IC95\% 23,6-38,8).

\section{Internação hospitalar}

Do total, 11,1\% (IC95\% 10,3-12,0) dos idosos foram internados ao menos uma vez no último ano. Dentre aqueles que não foram internados, 0,32\% (IC95\% 0,2-0,5) informaram ter necessitado de internação, conferindo uma prevalência de falta de acesso de 2,5\% (IC95\% 1,6-4,0) (Tabela 2). A maioria dos indivíduos foi internada pelo SUS (60,4\% - IC95\% 56,3-64,5), $32,1 \%$ (IC95\% 28,2-36,2) por convênio e 7,5\% (IC95\% 5,6-9,9) particular.

O tempo para conseguir a internação não diferiu entre as três vias de acesso, concentrando entre 0 a 3 dias de espera. Os idosos que internaram pelo SUS tiveram maior frequência de tempo de espera superior a 31 dias, entretanto, sem diferença estatística (Tabela 4).

O meio de transporte mais utilizado para se deslocar até o local de internação foi carro particular em todas as formas de financiamento, totalizando 56,1\% (IC95\% 52,2-59,9). Entretanto, os idosos que internaram pelo SUS utilizaram menos essa forma de transporte (47,2\% - IC95\% 42,2-52,3). Ambulância ou SAMU (Serviço de Atendimento Móvel de Urgência) foram mais utilizados pelos idosos que internaram pelo SUS (28,1\% - IC95\% 23,8-32).

Os usuários que internaram por via particular foram os que mais internaram fora do município de residência (48,2\% - IC95\% 33,8-62,9), enquanto aqueles que o fizeram via SUS foram os que menos saíram de sua cidade de residência para hospitalização (22,7\% - IC95\% 18,6-27,3), entretanto sem diferença em relação aos idosos que internaram via convênio (26,3\% - IC 95\% 20,4-33,2). A maior parte das pessoas necessitou 
Tabela 3. Características da utilização do atendimento médico. Brasil, 2009.

\begin{tabular}{|c|c|c|c|c|c|c|c|c|}
\hline & \multicolumn{2}{|c|}{$\begin{array}{c}\text { Convênio } \\
(840)\end{array}$} & \multicolumn{2}{|c|}{$\begin{array}{l}\text { Particular } \\
\quad(313)\end{array}$} & \multicolumn{2}{|c|}{ SUS (1865) } & \multicolumn{2}{|r|}{ Total } \\
\hline & $\%$ & 95\%CI & $\%$ & 95\%CI & $\%$ & 95\%CI & $\%$ & 95\%CI \\
\hline \multicolumn{9}{|c|}{ Foi encaminhado por algum serviço de saúde $(\mathrm{n}=3.006)$} \\
\hline Encaminhado & 10,3 & $8,3-12,6$ & 6,4 & $4,2-9,6$ & 13,8 & $12,0-15,9$ & 12,0 & $10,7-13,6$ \\
\hline Conta própria & 89,7 & $87,4-91,7$ & 93,6 & $90,4-95,8$ & 86,2 & $84,1-88,4$ & 88,0 & $86,5-89,3$ \\
\hline \multicolumn{9}{|c|}{ Que serviço encaminhou para consulta médica $(\mathrm{n}=364)$} \\
\hline Unidade de Saúde & 5,8 & $2,5-13,0$ & 13,6 & $4,4-35,0$ & 77,0 & $71,2-81,8$ & 56,3 & $50,7-61,8$ \\
\hline Ambulatório do hospital & 12,8 & $7,2-21,8$ & 13,6 & $4,4-35,0$ & 10,9 & $7,6-15,5$ & 11,5 & $8,6-15,3$ \\
\hline Consultório por convênio & 70,9 & $60,0-79,2$ & 9,1 & $2,3-30,2$ & 0,8 & $0,2-3,1$ & 17,9 & $14,1-22,3$ \\
\hline Consultório particular & 2,3 & $0,6-8,7$ & 59,1 & $38,0-77,3$ & 2,3 & $1,1-5,2$ & 5,8 & $3,8-8,7$ \\
\hline Outro & 8,1 & $3,9-16,2$ & 4,6 & $0,6-26,4$ & 9,0 & $6,0-13,2$ & 8,5 & $6,0-11,9$ \\
\hline \multicolumn{9}{|c|}{$\begin{array}{l}\text { Quanto tempo demorou até conseguir o atendimento } \\
(\mathrm{n}=2.965)\end{array}$} \\
\hline No mesmo dia & 46,9 & $43,0-50,8$ & 53,6 & $47,8-59,3$ & 50,7 & $47,8-53,6$ & 50,0 & $47,7-52,2$ \\
\hline 1 a 5 dias & 25,9 & $22,6-29,4$ & 27,9 & $23,1-33,4$ & 15,0 & $13,1-17,0$ & 19,4 & $17,7-21,1$ \\
\hline 6 a 15 dias & 17,9 & $15,2-21,0$ & 12,7 & $9,3-17,0$ & 13,8 & $12,1-15,6$ & 14,8 & $13,5-16,1$ \\
\hline 16 a 30 dias & 6,9 & $5,2-9,1$ & 3,3 & $1,8-5,9$ & 10,4 & $9,0-12,1$ & 8,7 & $7,6-9,9$ \\
\hline 31 a 60 dias & 1,0 & $0,4-2,4$ & 1,6 & $0,7-3,8$ & 3,3 & $2,5-4,4$ & 2,5 & $1,9-3,2$ \\
\hline Mais de 60 dias & 1,6 & $0,9-2,8$ & 1,0 & $0,3-2,8$ & 6,9 & $5,6-8,5$ & 4,8 & $3,9-5,9$ \\
\hline \multicolumn{9}{|c|}{$\begin{array}{l}\text { O local onde foi atendido pelo médico fica na cidade } \\
\text { onde mora }(n=3.010)\end{array}$} \\
\hline Não & 15,2 & $12,1-18,9$ & 24,6 & $19,6-30,4$ & 5,7 & $4,6-7,0$ & 10,3 & $8,9-11,9$ \\
\hline \multicolumn{9}{|c|}{$\begin{array}{l}\text { Tempo de transporte até o município para atendimento } \\
(\mathrm{n}=301)\end{array}$} \\
\hline 15 minutos ou menos & 2,4 & $0,8-7,3$ & 2,7 & $0,7-10,1$ & 9,9 & $5,4-17,6$ & 5,0 & $3,0-8,2$ \\
\hline 16 a 30 minutos & 27,2 & $18,8-37,7$ & 29,3 & $19,6-41,5$ & 20,8 & $13,4-30,8$ & 25,6 & $19,9-32,2$ \\
\hline 31 a 60 minutos & 35,2 & $26,0-48,0$ & 36,0 & $25,6-48,0$ & 31,7 & $23,0-41,9$ & 34,2 & $28,1-41,0$ \\
\hline 61 a 120 minutos & 23,2 & $16,3-31,9$ & 17,3 & $10,4-27,5$ & 20,8 & $13,3-31,1$ & 20,9 & $16,2-26,7$ \\
\hline Mais de 120 minutos & 12,0 & $6,7-20,6$ & 14,7 & $7,8-26,5$ & 16,8 & $10,4-26,0$ & 14,3 & $10,4-19,3$ \\
\hline \multicolumn{9}{|c|}{ Por que teve que ir para outra cidade $(n=308)$} \\
\hline Não tem médico & 12,5 & $7,4-19,5$ & 16,9 & $9,4-27,1$ & 11,7 & $6,6-19,7$ & 13,3 & $9,5-18,4$ \\
\hline Não atendem este tipo & 25,0 & $16,9-35,4$ & 27,3 & $18,7-38,0$ & 50,5 & $39,7-61,3$ & 34,1 & $27,8-41,0$ \\
\hline Não gosta do atendimento & 20,3 & $13,9-28,6$ & 26,0 & $16,9-37,6$ & 9,7 & $5,0-18,0$ & 18,2 & $14,0-23,3$ \\
\hline Não resolvem & 2,3 & $0,8-7,1$ & 13,0 & $7,3-22,2$ & 7,8 & $3,7-15,8$ & 6,8 & $4,4-10,4$ \\
\hline Outro motivo & 39,8 & $30,5-50,1$ & 16,9 & $9,7-27,8$ & 20,4 & $11,6-28,0$ & 27,6 & $22,3-33,2$ \\
\hline \multicolumn{9}{|c|}{ Como foi até o local de atendimento médico $(\mathrm{n}=3.002)$} \\
\hline Caminhando & 13,6 & $11,0-16,8$ & 13,1 & $9,8-17,4$ & 57,9 & $55,2-60,7$ & 40,9 & $38,7-43,2$ \\
\hline Ônibus/lotação/Van/Kombi & 26,4 & $22,9-30,2$ & 25,9 & $21,2-31,1$ & 16,7 & $14,7-18,9$ & 20,4 & $18,6-22,3$ \\
\hline Ambulância/ SAMU & 0,8 & $0,4-1,8$ & 1,3 & $0,5-3,4$ & 2,7 & $2,0-3,6$ & 2,0 & $1,5-2,7$ \\
\hline Carro particular & 53,8 & $49,8-57,7$ & 53,7 & $47,8-59,5$ & 16,8 & $15,1-18,7$ & 31,0 & $28,8-33,2$ \\
\hline Taxi & 5,0 & $3,7-6,8$ & 5,8 & $3,5-9,3$ & 2,1 & $1,5-2,9$ & 3,3 & $2,6-4,1$ \\
\hline Outra maneira & 1,3 & $0,6-2,7$ & 1,9 & $0,9-4,2$ & 1,8 & $1,3-2,5$ & 1,7 & $1,3-2,3$ \\
\hline \multicolumn{9}{|c|}{$\begin{array}{l}\text { Gastou algum dinheiro para ir até o local do atend. } \\
\text { médico }(n=3.004)\end{array}$} \\
\hline Sim & 20,8 & $17,8-24,1$ & 28,5 & $23,3-34,4$ & 9,5 & $8,1-11,2$ & 14,7 & $13,2-16,2$ \\
\hline \multicolumn{9}{|c|}{$\begin{array}{l}\text { Saiu da consulta com encaminhamento para outro local } \\
(\mathrm{n}=3.003)\end{array}$} \\
\hline Sim & 4,8 & $3,5-6,6$ & 5,1 & $3,2-8,2$ & 8,2 & $7,0-9,7$ & 6,9 & $6,0-8,0$ \\
\hline \multicolumn{9}{|c|}{$\begin{array}{l}\text { Conseguiu atendimento no local para onde foi } \\
\text { encaminhado }(n=208)\end{array}$} \\
\hline Sim & 72,5 & $56,8-84,1$ & 87,5 & $61,0-96,9$ & 61,2 & $52,8-67,0$ & 65,4 & $58,4-71,8$ \\
\hline
\end{tabular}


Tabela 4. Características da utilização da Internação hospitalar. Brasil, 2009.

\begin{tabular}{|c|c|c|c|c|c|c|c|c|}
\hline & \multicolumn{2}{|c|}{$\begin{array}{l}\text { Convênio } \\
(232)\end{array}$} & \multicolumn{2}{|c|}{ Particular (54) } & \multicolumn{2}{|c|}{ SUS (437) } & \multicolumn{2}{|c|}{ Total } \\
\hline & $\%$ & 95\%CI & $\%$ & 95\%CI & $\%$ & 95\%CI & $\%$ & $95 \% \mathrm{CI}$ \\
\hline \multicolumn{9}{|l|}{$\begin{array}{l}\text { Que serviço encaminhou para internação } \\
(\mathrm{n}=718)\end{array}$} \\
\hline Unidade de saúde & 2,6 & $1,2-5,7$ & 7,4 & $2,8-18,1$ & 31,4 & $27,0-36,2$ & 20,3 & $17,3-23,7$ \\
\hline Pronto-socorro do SUS & 4,8 & $2,7-8,4$ & 3,7 & $1,0-13,2$ & 27,7 & $23,3-32,4$ & 18,5 & $15,7-21,8$ \\
\hline Ambulatório do hospital & 14,7 & $10,4-20,4$ & 37,0 & $23,4-53,1$ & 27,7 & $23,3-32,4$ & 24,2 & $20,7-28,2$ \\
\hline Consultório por convênio & 63,6 & $57,0-69,8$ & 5,6 & $1,8-16,1$ & 1,2 & $0,5-2,8$ & 21,6 & $18,4-25,2$ \\
\hline Consultório particular & 4,3 & $2,4-7,8$ & 42,6 & $29,3-57,0$ & 4,6 & $2,0-7,0$ & 7,4 & $5,7-9,5$ \\
\hline Outro & 10,0 & $6,8-14,5$ & 3,7 & $0,9-13,5$ & 7,4 & $5,2-10,4$ & 7,9 & $6,2-10,2$ \\
\hline \multicolumn{9}{|l|}{$\begin{array}{l}\text { Quanto tempo demorou até conseguir } \\
\text { internar }(n=710)\end{array}$} \\
\hline 0 a 3 dias & 86,1 & $80,8-90,1$ & 81,5 & $69,5-89,5$ & 82,6 & $78,8-85,9$ & 83,7 & $80,7-86,2$ \\
\hline 4 a 10 dias & 7,0 & $4,4-11,0$ & 9,3 & $4,0-20,2$ & 3,5 & $2,1-5,9$ & 5,1 & $3,6-7,0$ \\
\hline 11 a 20 dias & 1,7 & $0,7-4,5$ & 7,4 & $2,8-18,1$ & 3,5 & $2,1-5,9$ & 3,2 & $2,1-4,9$ \\
\hline 21 a 30 dias & 2,6 & $1,2-5,6$ & 0 & -- & 3,1 & $1,8-5,2$ & 2,7 & $1,7-4,1$ \\
\hline 31dias e mais & 2,6 & $1,2-5,6$ & 1,9 & $0,3-11,8$ & 7,3 & $5,0-10,0$ & 5,4 & $4,0-7,2$ \\
\hline \multicolumn{9}{|l|}{ Internou na cidade onde mora $(\mathrm{n}=723)$} \\
\hline Não & 26,3 & $20,4-33,2$ & 48,2 & $33,8-62,9$ & 22,7 & $18,6-27,3$ & 25,7 & $22,1-29,8$ \\
\hline \multicolumn{9}{|l|}{$\begin{array}{l}\text { Tempo de transporte até o município } \\
\text { para atendimento }(n=181)\end{array}$} \\
\hline 15 minutos ou menos & 6,7 & $2,5-16,4$ & 26,9 & $13,5-46,5$ & 14,7 & $8,2-25,1$ & 13,8 & $9,0-20,7$ \\
\hline 16 a 30 minutos & 20,0 & $11,4-32,8$ & 19,2 & $8,0-39,4$ & 21,1 & $13,6-31,2$ & 20,4 & $14,7-27,8$ \\
\hline 31 a 60 minutos & 38,3 & $25,9-52,5$ & 19,2 & $8,8-37,2$ & 22,1 & $15,0-31,3$ & 27,1 & $20,7-34,5$ \\
\hline 61 a 120 minutos & 23,3 & $13,8-36,6$ & 23,1 & $9,9-45,0$ & 21,1 & $13,7-30,9$ & 22,1 & $16,2-29,4$ \\
\hline Mais de 120 minutos & 11,7 & $5,7-22,5$ & 11,5 & $3,7-30,7$ & 21,1 & $13,4-31,6$ & 16,6 & $11,0-24,3$ \\
\hline \multicolumn{9}{|l|}{$\begin{array}{l}\text { Por que teve que ir para outra cidade } \\
(\mathrm{n}=186)\end{array}$} \\
\hline Não tem hospital & 9,8 & $3,9-22,6$ & 11,5 & $2,8-29,9$ & 21,2 & $13,0-32,7$ & 16,1 & $10,4-24,2$ \\
\hline Não tem médico & 1,6 & $0,2-11,0$ & 3,9 & $0,5-23,4$ & 8,1 & $4,0-15,5$ & 5,4 & $2,9-9,8$ \\
\hline Não atendem este tipo de problema & 27,9 & $17,8-40,8$ & 50,0 & $30,8-69,2$ & 45,6 & $35,6-55,7$ & 40,3 & $33,2-47,9$ \\
\hline Não gosta do atendimento & 24,6 & $15,3-37,0$ & 15,4 & $5,7-35,4$ & 9,1 & $4,8-16,6$ & 15,1 & $10,6-21,0$ \\
\hline Outro motivo & 36,1 & $24,6-49,4$ & 19,2 & $8,0-39,6$ & 16,2 & $10,0-25,4$ & 23,1 & $17,1-30,4$ \\
\hline \multicolumn{9}{|l|}{$\begin{array}{l}\text { Como foi para o hospital para internar } \\
(\mathrm{n}=720)\end{array}$} \\
\hline Caminhando & 5,2 & $2,7-9,2$ & 7,4 & $2,4-21,0$ & 5,3 & $3,4-8,2$ & 5,4 & $3,8-7,6$ \\
\hline Ônibus/lotação/Van/Kombi & 8,2 & $5,1-12,9$ & 3,7 & $0,9-13,8$ & 9,5 & $6,9-12,8$ & 8,6 & $6,7-11,0$ \\
\hline Ambulância/ SAMU & 12,1 & $8,6-16,6$ & 1,9 & $0,3-12,2$ & 28,1 & $23,8-32,9$ & 21,0 & $18,0-24,3$ \\
\hline Carro particular & 67,2 & $61,0-72,9$ & 79,6 & $66,1-88,7$ & 47,2 & $42,2-52,3$ & 56,1 & $52,2-59,9$ \\
\hline Taxi & 5,6 & $3,3-9,5$ & 3,7 & $0,9-13,8$ & 6,0 & $4,1-8,8$ & 5,7 & $4,2-7,8$ \\
\hline Outra maneira & 3,5 & $1,8-6,7$ & 9,3 & $3,9-20,5$ & 5,1 & $3,4-7,5$ & 4,9 & $3,5-6,8$ \\
\hline \multicolumn{9}{|l|}{$\begin{array}{l}\text { Foi necessário gastar algum dinheiro para } \\
\text { ir até o hospital }(n=721)\end{array}$} \\
\hline Sim & 22,0 & $16,8-28,2$ & 25,9 & $16-39,2$ & 18,9 & $15,0-23,4$ & 20,4 & $17,3-23,9$ \\
\hline \multicolumn{9}{|l|}{$\begin{array}{l}\text { Saiu do hospital encaminhado para } \\
\text { consulta de revisão? }(n=712)\end{array}$} \\
\hline Sim & 54,4 & $47,4-61,2$ & 63,0 & $49,9-74,4$ & 37,9 & $33,0-43,0$ & 45,1 & $41,0-49,2$ \\
\hline
\end{tabular}


se deslocar pelo fato do município ser desprovido do tipo de atendimento hospitalar requerido $(40,3 \%$ - IC95\% 33,2-47,9). Apesar de haver sobreposição dos intervalos de confiança, os usuários do SUS foram os que menos alegaram ter internado em outro município por não gostar do atendimento.

Aproximadamente um terço dos respondentes referiu tempo de deslocamento de 31 a $60 \mathrm{mi}-$ nutos até o município de internação. Os usuários do SUS foram aqueles que mais citaram tempo de deslocamento superior a 120 minutos $(21,1 \%$ - IC95\% 13,4-31,6), mas sem diferença entre as formas de financiamento.

Do total de respondentes, a menor frequência de encaminhamento para consulta de revisão foi observada entre os que internaram pelo SUS (37,9\% - IC95\% 33,0-43,0), valor significativamente menor do que o observado para usuários de convênio e particular.

\section{Pronto-socorro}

A prevalência de utilização de pronto-socorro nos 12 meses anteriores à entrevista foi de $16,5 \%$ (IC95\% 15,3-17,6) e 0,4\% (IC95\% 0,3$0,6)$ dos que não utilizaram referiram ter necessitado, conferindo uma falta de acesso de 2,1\% (IC95\% 1,4-3,1) (Tabela 2). A maior parte dos atendimentos foi pelo SUS (85,4\% - IC95\% 82,7$87,7)$. Grande parte dos idosos foi ao serviço por conta própria e aguardou de 1 a 15 minutos para ser atendido, sem diferença em relação ao tempo de espera entre convênio (32,4\% - IC95\% 25,3$40,4)$, particular $(35,7 \%$ - IC95\% 15,6-62,5) e SUS $(35,7 \%$ - IC95\% 32,5-39,0) (Tabela 5).

Apenas 5,4\% (IC95\% 4,0-7,4) dos idosos atendidos pelo SUS necessitaram sair do município de residência, contra 13,0\% (IC95\% 7,9-20,8) dos que utilizaram via convênio e 14,3\% (IC95\% $3,6-42,8)$ dos atendidos por via particular. Nas três formas de financiamento, o principal motivo de ter que se deslocar até outro município foi não haver pronto-socorro no local. Mais da metade dos idosos atendidos fora do município de residência referiram tempo de deslocamento inferior a 30 minutos.

Não houve diferença entre as três formas de financiamento em relação ao recebimento de encaminhamento para consulta de revisão e realização da mesma. A prevalência de encaminhamento foi de 21,6\% (IC95\% 15,4-29,4) entre os idosos atendidos pelo convênio, 21,4\% (IC95\% $7,0-49,6)$ entre os atendidos via particular e $14 \%$ (IC95\% 11,7-16,6) entre os atendidos pelo SUS.

\section{Discussão}

A prevalência de falta de acesso reportada foi relativamente baixa nos três tipos de serviços, alcançando 2,5\% nos serviços de internação, $2,1 \%$ em pronto-socorro, e $0,6 \%$ no atendimento médico. A baixa falta de acesso identificada é convergente com a Pesquisa Nacional por Amostra de Domicílios (PNAD) e Pesquisa Nacional de Saúde (PNS). Em 2003 a PNAD ${ }^{12}$ identificou $1,2 \%$ da população maior de 65 anos sem êxito na primeira ou última tentativa de atendimento, em um período de duas semanas, alcançando a prevalência de $1,8 \%$ em $2008^{13}$. De acordo com a PNS 2013 a falta de acesso em idosos maiores que 60 anos correspondeu a $3,2 \%$ considerando a primeira tentativa num período de duas semanas ${ }^{14}$. A diferença entre a taxa encontrada no presente estudo e na PNAD deve ser ponderada considerando a diferença do período recordatório adotado nos estudos. Neste trabalho o período foi maior que o adotado pela PNAD. Dessa forma, os usuários tiveram mais tempo para realizar tentativas até conseguir atendimento resultando em menor falta de acesso. Além disso, a PNAD se restringia à primeira ou última tentativa e a PNS à primeira tentativa. Com relação à utilização de consulta médica, a taxa encontrada (46\%) foi proporcionalmente semelhante à de outros estudos nacionais, levando em consideração diferenças no período recordatório. Na PNS de 2013 a prevalência de consulta médica nos últimos 12 meses foi $83,5 \%{ }^{2}$.

A baixa taxa de falta de acesso no atendimento médico pode ser resultado da melhoria da cobertura de serviços de Atenção Primária à Saúde (APS) no país que trata os idosos como grupo prioritário nas ações de saúde. Além disso, $\mathrm{o}$ atendimento a pessoas com hipertensão e diabetes também é prioridade nesse nível de atenção, doenças com alta prevalência na terceira idade $^{15,16}$. Estudo realizado em Porto Alegre demonstrou que os serviços de Atenção Primária foram utilizados por $49,7 \%$ dos idosos nos seis meses anteriores a entrevista ${ }^{17}$. À semelhança de outros achados no país ${ }^{18,19}$, o referido estudo também observou a associação entre ter doença crônica e utilizar serviço de APS.

A população idosa parece ter mais acesso aos serviços de saúde quando comparada à população adulta. Estudo realizado com base no mesmo inquérito populacional utilizado neste trabalho encontrou, na população de 20 a 59 anos, uma taxa de falta de acesso à consulta médica de $2,1 \%$, ou seja, 3,5 vezes maior do que a observada neste 
Tabela 5. Características da utilização do atendimento em pronto-socorro. Brasil, 2009.

\begin{tabular}{|c|c|c|c|c|c|c|c|c|}
\hline & \multicolumn{2}{|c|}{ Convênio (139) } & \multicolumn{2}{|c|}{ Particular (14) } & \multicolumn{2}{|c|}{ SUS (896) } & \multicolumn{2}{|c|}{ Total } \\
\hline & $\%$ & 95\%CI & $\%$ & 95\%CI & $\%$ & 95\%CI & $\%$ & 95\%CI \\
\hline \multicolumn{9}{|l|}{$\begin{array}{l}\text { Foi encaminhado por algum serviço de } \\
\text { saúde }(n=1.049)\end{array}$} \\
\hline Encaminhado & 3,6 & $1,5-8,2$ & 0 & -- & 4,6 & $3,3-6,3$ & 4,4 & $3,3-5,9$ \\
\hline Conta própria & 65,5 & $56,7-73,3$ & 71,4 & $43,8-88,9$ & 61,5 & $57,9-65,0$ & 62,2 & $58,8-65,4$ \\
\hline Levado & 30,9 & $23,6-39,4$ & 28,6 & $11,1-56,2$ & 33,9 & $30,6-37,4$ & 33,5 & $30,3-36,8$ \\
\hline \multicolumn{9}{|l|}{$\begin{array}{l}\text { Quanto tempo demorou até conseguir } \\
\text { o atendimento }(\mathrm{n}=1.015)\end{array}$} \\
\hline Entrou direto & 17,7 & $12,0-25,3$ & 28,6 & $11,1-56,2$ & 13,3 & $11,2-15,8$ & 14,1 & $12,0-16,5$ \\
\hline $1 \mathrm{a} 15 \mathrm{~min}$ & 32,4 & $25,3-40,4$ & 35,7 & $15,6-62,5$ & 35,7 & $32,5-39,0$ & 35,3 & $32,3-38,3$ \\
\hline 16 a $30 \mathrm{~min}$ & 25,0 & $17,8-33,6$ & 14,3 & $3,6-42,8$ & 25,0 & $22,3-27,9$ & 24,9 & $22,3-27,6$ \\
\hline 31 a $60 \mathrm{~min}$ & 16,2 & $11,1-23,1$ & 14,3 & $3,6-42,8$ & 10,1 & $8,3-12,2$ & 10,9 & $9,2-12,4$ \\
\hline 61 a $120 \mathrm{~min}$ & 6,6 & $3,5-12,2$ & 0 & -- & 10,1 & $8,3-12,2$ & 9,5 & $7,9-11,6$ \\
\hline Mais de $120 \mathrm{~min}$ & 2,2 & $0,7-6,6$ & 7,1 & $1,0-37,2$ & 5,9 & $4,4-7,9$ & 5,4 & $4,1-7,2$ \\
\hline \multicolumn{9}{|l|}{$\begin{array}{l}\text { O pronto-socorro fica na cidade onde } \\
\text { mora }(n=1.048)\end{array}$} \\
\hline Não & 13,0 & $7,9-20,8$ & 14,3 & $3,6-42,8$ & 5,4 & $4,0-7,4$ & 6,6 & $5,0-8,6$ \\
\hline \multicolumn{9}{|l|}{$\begin{array}{l}\text { Tempo de transporte até o município } \\
\text { para atendimento }(n=64)\end{array}$} \\
\hline 15 minutos ou menos & 16,7 & $5,3-41,6$ & 50,0 & $5,9-94,2$ & 38,4 & $26,0-53,0$ & 32,8 & $22,9-44,5$ \\
\hline 16 a 30 minutos & 33,3 & $14,1-60,3$ & 50,0 & $5,9-94,2$ & 31,8 & $20,0-46,8$ & 32,8 & $22,4-45,2$ \\
\hline 31 a 60 minutos & 44,4 & $22,2-69,2$ & 0 & -- & 18,2 & $9,6-31,9$ & 25,0 & $15,6-37,6$ \\
\hline Mais de 60 minutos & 5,6 & $0,8-31,1$ & 0 & -- & 11,4 & $4,7-24,8$ & 9,4 & $4,2-19,6$ \\
\hline \multicolumn{9}{|l|}{$\begin{array}{l}\text { Por que teve que ir para outra cidade } \\
(n=69)\end{array}$} \\
\hline Não tem Pronto-socorro & 27,8 & $11,3-53,8$ & 50,0 & $5,4-94,6$ & 38,0 & $24,5-53,6$ & 35,7 & $24,1-49,2$ \\
\hline Não tem médico & 5,6 & $0,7-32,3$ & 0 & -- & 2,0 & $0,3-13,2$ & 2,9 & $0,7-10,8$ \\
\hline Não atendem esse tipo de problema & 11,1 & $2,6-36,7$ & 0 & -- & 10,0 & $4,1-22,3$ & 10,0 & $4,7-20,0$ \\
\hline Não gosta do atendimento & 8,3 & $0,7-32,3$ & 0 & -- & 10,0 & $4,2-21,8$ & 8,6 & $3,9-17,8$ \\
\hline Outro motivo & 50,0 & $5,4-94,6$ & 50,0 & $5,4-94,6$ & 40,0 & $26,8-54,8$ & 42,9 & $30,3-56,4$ \\
\hline \multicolumn{9}{|l|}{$\begin{array}{l}\text { Como foi para o Pronto-Socorro } \\
(\mathrm{n}=1.045)\end{array}$} \\
\hline Caminhando & 5,1 & $2,5-10,2$ & 14,3 & $3,6-42,8$ & 11,2 & $9,1-13,7$ & 10,4 & $8,5-12,7$ \\
\hline Ônibus/lotação/Van/Kombi & 2,2 & $0,7-6,5$ & 0 & -- & 11,3 & $9,0-14,1$ & 10,0 & $8,0-12,4$ \\
\hline Ambulância/ SAMU & 8,0 & $4,6-13,5$ & 14,3 & $3,6-42,8$ & 14,8 & $12,4-17,6$ & 13,9 & $11,7-16,4$ \\
\hline Carro particular & 74,6 & $66,7-81,2$ & 78,6 & $50,5-100,0$ & 51,5 & $47,9-55,1$ & 54,9 & $51,5-58,3$ \\
\hline Taxi & 7,3 & $3,7-13,7$ & 0 & -- & 5,2 & $3,9-6,9$ & 5,4 & $4,1-7,0$ \\
\hline Outra maneira & 2,8 & $0,7-6,5$ & 0 & -- & 6,5 & $5,0-8,5$ & 5,8 & $4,5-7,6$ \\
\hline \multicolumn{9}{|l|}{$\begin{array}{l}\text { Gastou algum dinheiro em transporte } \\
\text { para ir até o Pronto-socorro }(n=1.049)\end{array}$} \\
\hline Sim & 21,6 & $15,3-29,5$ & 28,6 & $11,1-56,2$ & 16,5 & $13,9-19,5$ & 17,4 & $14,9-20,2$ \\
\hline \multicolumn{9}{|l|}{$\begin{array}{l}\text { Saiu do PS encaminhado para consulta } \\
\text { de revisão? }(n=1.042)\end{array}$} \\
\hline Sim & 21,6 & $15,4-29,4$ & 21,4 & $7,0-49,6$ & 14,0 & $11,7-16,6$ & 15,1 & $12,9-17,5$ \\
\hline \multicolumn{9}{|l|}{ Fez a consulta de revisão $(n=157)$} \\
\hline Sim & 93,3 & $77,4-98,3$ & 100,0 & -- & 75,8 & $67,7-82,4$ & 79,6 & $72,7-85,2$ \\
\hline
\end{tabular}


estudo entre idosos ${ }^{20}$. A PNAD demonstrou, para os adultos, taxas de falta de acesso de 2,2\% e 2,8\% em 2003 e $2008^{12,13}$, respectivamente. Em relação à internação também foi observada menor taxa de falta de acesso comparada aos adultos. No estudo de Dilélio et al. ${ }^{20}$ foi de $3 \%$, enquanto neste estudo foi de $2,5 \%$. No atendimento em prontosocorro as taxas foram idênticas para adultos e idosos (2,1\%).

Indivíduos idosos também utilizam mais os serviços de saúde. Em adultos a taxa de utilização de consulta médica foi de $34,6 \%$ de acordo com estudo de Dilélio et al. ${ }^{21} \mathrm{e}$ a taxa de internação na população de 20 a 64 anos foi de 7,4\% segundo a PNAD 2008. A maior utilização de serviços de saúde pelos idosos pode se dever ao fato de que essa população é acometida com maior frequência por condições crônicas de saúde e incapacidades, inclusive manifestando simultaneamente várias morbidades ${ }^{22}$, tendendo a aumentar a necessidade de atenção à saúde ${ }^{2,23,24}$. Além disso, por apresentarem piores condições de saúde que outros grupos etários, os idosos tendem a procurar mais os serviços de saúde, e um estudo recente demonstrou que a procura tem aumentando nesse grupo populacional no Brasil ${ }^{14}$.

Políticas com foco no idoso também podem estar contribuindo para essa realidade, promovendo acessibilidade e atendimento prioritário à pessoa idosa ${ }^{25}$. Além disso, o SUS por sua natureza pública e gratuita facilita o acesso, especialmente em caso de busca persistente pelo serviço, que pode ser mais frequente em pessoas já aposentadas, sem inserção tão presente em atividades ocupacionais.

A proporção de internação de idosos nos últimos 12 meses observada neste estudo de $11,1 \%$ foi semelhante à da PNAD de 2008, que evidenciou prevalência anual de $13,5 \%{ }^{13}$, e com a PNS que foi de $10,2 \%^{2}$. A taxa anual de utilização de pronto-socorro neste estudo foi semelhante à encontrada na Espanha $(14,7 \%)^{26}$. São escassos estudos sobre utilização de serviços de emergência por idosos no Brasil.

O presente estudo demonstrou grande utilização de serviços de saúde ofertados pelo SUS, especialmente em pronto-socorro. Idosos encontram dificuldades em contratar planos de saúde em virtude das altas mensalidades e acabam dependendo dos serviços do SUS. Segundo pesquisa realizada pelo Idec, a média dos preços dos planos de saúde individuais/familiares mais baratos ofertados na cidade de São Paulo compromete cerca de $40 \%$ da renda mensal dos idosos ${ }^{27}$. De acordo com a ANS, menos de $20 \%$ da popu- lação idosa que recebe até 1 salário mínimo possui planos de saúde. Já na faixa correspondente a uma renda superior a cinco salários mínimos, esse percentual é em torno de $85 \%{ }^{28}$.

O SUS demonstrou bom desempenho não apenas no acesso e na utilização de serviços, mas também no tempo para conseguir atendimento, não diferindo substancialmente do sistema privado. Em torno de metade dos idosos conseguiu consultar o médico no mesmo dia e 7,3\% demoraram mais de 30 dias. Para tempo de espera maior, o SUS se destacou neste estudo, embora a diferença não seja muito expressiva. Além disso, o estudo demonstrou que a grande maioria dos atendimentos médicos foi realizada na cidade de residência do idoso, especialmente via SUS. Esses fatos podem ser justificados pela maior capilaridade da APS no sistema público, garantindo acesso e atendimento médico mais próximo a residência. $\mathrm{O}$ grande percentual de idosos que foram caminhando para obter atendimento médico pelo SUS, reforça a proximidade do serviço. De acordo com estudo de Rodrigues et al. ${ }^{19}$ realizado nas regiões sul e nordeste do Brasil, aproximadamente 50\% dos idosos que consultaram em nível ambulatorial utilizaram a unidade básica de saúde de abrangência. No estudo de Paskulin et al. ${ }^{17}$ a grande utilização dos serviços de APS no SUS, foi motivada por sua localização próxima a residência, além da qualidade e "gratuidade".

No uso de serviços de internação houve maior percentual de atendimentos em outro município, com destaque para aqueles que o fizeram por desembolso direto, não havendo diferença entre SUS e planos privados. Na perspectiva das redes de atenção e da regionalização na saúde, os serviços de Atenção Primária devem ser o mais amplamente distribuído no território, devendo ser ofertados em todos os municípios, e os serviços mais especializados, como internação, podem ser concentrados em determinada localidade. A concentração geográfica com ampliação da cobertura populacional dos serviços mais complexos é uma forma de otimizar os recursos disponíveis no território ${ }^{29} \mathrm{e}$ obter maior racionalidade no uso dos serviços, favorecendo o acesso a todos que deles necessitam.

O fato de não haver diferença entre o SUS e planos de saúde na internação em municípios diferentes da residência pode demonstrar o quão imbricado estão o público e o privado na oferta de serviços de saúde hospitalares, existindo em determinadas regiões uma só rede para ambos segmentos ${ }^{30,31}$. Já as pessoas que realizam desembolso direto podem fazer escolhas mais livre- 
mente e muitas vezes optam por utilizar serviços em municípios mais distantes.

A justificativa mais frequente dos idosos que utilizaram consulta médica por desembolso direto em outro município foi por não gostar do atendimento, enquanto no SUS e plano, por não haver o serviço no município. Esse fato ao mesmo tempo em que pode refletir a capacidade de escolha do prestador pelo cliente, pode refletir também a satisfação com o serviço ofertado. Somente $9,7 \%$ dos idosos que utilizaram consulta médica pelo SUS relataram terem feito em outro município por não gostar do atendimento. Outros estudos também têm demostrado grande satisfação dos idosos com serviços de consulta médica no SUS ${ }^{32,33}$.

O tempo de transporte gasto por idosos que consultaram ou internaram fora do município de residência se concentrou entre 31 a 60 minutos. Para os idosos que utilizaram pronto-socorro o tempo de deslocamento foi ainda menor (menos de 30 minutos) indicando que possivelmente esses serviços foram ofertados dentro de sua região de saúde, estratégia fundamental para ampliar e assegurar o acesso da população aos serviços de saúde ${ }^{34}$.

Os idosos atendidos pelo SUS foram os que menos relataram gasto de dinheiro para ir até o local do atendimento nos três tipos de serviço, entretanto com diferença significativa entre as formas de financiamento somente na consulta médica. $\mathrm{O}$ mesmo resultado foi encontrado para os adultos no estudo de Dilélio et al. ${ }^{21}$. A responsabilização do sistema de saúde em garantir o transporte até o local do atendimento é fundamental para assegurar o acesso universal e igualitário aos cidadãos, especialmente quando se trata de idosos com dificuldade de locomoção.

Poucos idosos foram encaminhados para atendimento em outro local, sugerindo resolutividade da consulta médica tanto no público quanto no privado, apesar da diferença encontrada entre SUS e plano de saúde. Resultado semelhante foi encontrado por Dilélio et al. ${ }^{21}$ entre adultos, em seu estudo 7,1\% dos entrevistados receberam encaminhamento, sendo mais frequente entre os atendidos pelo SUS (8,7\%). A maior frequência de encaminhamentos no SUS pode ser justificada pelo fluxo de encaminhamento pré-estabelecido entre os níveis de atenção no sistema público ${ }^{35}$. No privado é comum que os usuários recorram diretamente ao especialista.

Quando se avalia a continuidade do cuidado após atendimento em pronto-socorro e internação observam-se falhas. Tanto no público quanto no privado foi baixa a frequência de encaminhados para consulta de revisão após atendimento em pronto-socorro. Pouco menos da metade dos entrevistados tiveram alta da internação com encaminhamento para consulta de revisão. A menor frequência observada no SUS evidencia a fragmentação dos serviços e precariedade na articulação com a Atenção Primária à Saúde. Estudo de Dilélio et al. ${ }^{20}$ encontrou prevalência de $14,9 \%$ de encaminhamento para consulta de revisão após atendimento em pronto-socorro e $57,1 \%$ após alta hospitalar entre adultos brasileiros.

Neste estudo foi adotada a prevalência de falta de acesso como uma forma de mensuração de acesso. Muitos estudos adotam a utilização como proxy de acesso aos serviços de saúde. Entretanto, alguns autores defendem que acesso não equivale à simples utilização do serviço de saúde ${ }^{2,6,36}$. A utilização pode ser compreendida como a evidência de que o acesso foi alcançado. Entretanto, as taxas de utilização não permitem determinar o grau em que os serviços não foram usados, embora tenham sido necessários ${ }^{6}$. No uso de serviços de saúde, as populações que não conseguiram atendimento (falta de acesso) e aquelas que não precisaram de serviços de saúde, são consideradas em uma mesma categoria - não uso de serviços de saúde. Assim, a mensuração da falta de acesso busca evidenciar em que medida a demanda está sendo satisfeita ou não.

Uma fragilidade a ser destacada neste estudo foi a abordagem adotada que não permitiu mensurar as tentativas despendidas pelo idoso para conseguir o atendimento. Dessa forma, a medida de falta de acesso não considera as buscas fracassadas anteriores ao sucesso. Isso deve ser ponderado ao contrastar com as maiores prevalências encontradas na PNAD, que considera a primeira ou última tentativa.

Outra limitação foi a não inclusão de idosos institucionalizados sendo possível que as estimativas fossem diferentes se esses indivíduos fossem considerados. Além disso, pelo desenho do processo de amostragem, que buscou representatividade dos municípios brasileiros considerando o porte, não se alcançou a representatividade de todas as unidades da federação.

O presente estudo aponta para avanços no sistema público de saúde brasileiro na assistência a pessoa idosa, apesar das deficiências ainda existentes. A grande utilização do SUS em todos os tipos de atendimento reforça sua relevância para a universalidade da atenção à saúde no Brasil, demonstrando que é um sistema de saúde que pro- 
tege o idoso do risco financeiro, garantindo mais equidade e cidadania. Os achados demonstraram poucas diferenças do SUS em relação ao privado no que tange ao uso, tempo de espera e resolutividade. Aspectos positivos do SUS em relação ao privado foram maior número de atendimentos médicos realizados na cidade de residência do idoso e menor frequência de dinheiro gasto para se deslocar até o local de atendimento. O privado se destaca quando observamos a baixa frequência de tempo de espera mais longo e maior frequência de encaminhamentos para consulta de revisão após internação.

Tanto no sistema público quanto no privado faz-se necessário maior articulação dos níveis de atenção com vistas a oferecer continuidade do cuidado na perspectiva da integralidade da atenção à saúde da pessoa idosa.

\section{Colaboradores}

APSC Almeida participou na concepção do artigo, escrita da introdução, metodologia, realização da análise dos dados, redação dos resultados e discussão, e revisão final. BP Nunes participou na concepção do artigo, realização da análise dos dados, redação dos resultados e discussão, e revisão final. SMS Duro participou na concepção do artigo, na realização da análise dos dados, redação dos resultados e discussão, e revisão final. RCD Lima participou na concepção do artigo, redação dos resultados e discussão, e revisão final. LA Facchini participou na concepção do artigo, realização da análise dos dados, redação dos resultados e discussão, e revisão final. 


\section{Referências}

1. Veras R, Parahyba MI. O anacronismo dos modelos assistenciais para os idosos na área da saúde: desafios para o setor privado. Cad Saude Publ 2007; 23(10):2479-2489.

2. Stopa SR, Malta DC, Monteiro CN, Szwarcwald CL, Goldbaum M, Cesar CLG. Acesso e uso de serviços de saúde pela população brasileira, Pesquisa Nacional de Saúde 2013. Rev Saude Publica 2017; 51(Supl 1):3s.

3. Facchini LA, Nunes BP, Duro SMS, Thumé E, Siqueira F, Fripp JC, Fass AG, Tomasi E. Iniquidades socioeconômicas no acesso e na qualidade da atenção ao diabetes entre idosos brasileiros. In: Conselho Nacional de Secretários de Saúde (CONASS). CONASS Debate: a crise contemporânea dos modelos de atenção à saúde. Brasília: CONASS; 2014. p. 88-106.

4. Travassos C, Martins M. Uma revisão sobre os conceitos de acesso e utilização de serviços de saúde. Cad Saude Publica 2004; 20(Supl. 2):S190-S198.

5. Araújo MEA, Silva MT, Andrade KRC, Galvão TF, Pereira MG. Prevalência de utilização de serviços de saúde no Brasil: revisão sistemática e metanálise. Epidemiol Serv Saude 2017; 26(3):589-604.

6. Nunes BP, Thumé E, Tomasi E, Duro SMS, Facchini LA. Desigualdades socioeconômicas no acesso e qualidade da atenção nos serviços de saúde. Rev Saude Publica 2014; 48(6):968-976.

7. Oliver A, Mossialos E. Equity of access to health care: outlining the foundations for action. J Epidemiol Community Health 2004; 58(8):655-658.

8. Bahia L. A Démarche do Privado e Público no Sistema de Atenção à Saúde no Brasil em Tempos de Democracia e Ajuste Fiscal, 1988-2008. In: Lima JCF, Matta GC, organizadores. Estado, Sociedade e Formação Profissional em Saúde: Contradições e desafios em 20 anos de SUS. Rio de Janeiro: Editora Fiocruz; 2008. p. 123-185.

9. Santos IS, Ugá MAD, Porto SM. O mix público-privado no Sistema de Saúde Brasileiro: Financiamento, oferta e utilização dos serviços de saúde. Cien Saude Colet 2008; 13(5):1431-1440.

10. Cabral ALLV, Martinez-Hemáez A, Andrade EIG, Cherchiglia ML. Itinerários terapêuticos: o estado da arte da produção científica no Brasil. Cien Saude Colet 2011; 16(11):4433-4442.

11. Green LA, Fryer GE, Jr. Yawn BP, Lanier D, Dovey SM. The ecology of medical care revisited. $N$ Engl J Med 2001; 344(26):2021-2025

12. Instituto Brasileiro de Geografia e Estatística (IBGE). Pesquisa Nacional por Amostra de Domicílios (PNAD): acesso e utilização dos serviços de saúde 2003. Rio de Janeiro: IBGE; 2005.

13. Instituto Brasileiro de Geografia e Estatística (IBGE). Pesquisa Nacional por Amostra de Domicílios. Um Panorama da saúde no Brasil: acesso e utilização dos serviços, condições de saúde e fatores de risco e proteção à saúde: 2008. Rio de Janeiro: IBGE; 2010.

14. Nunes BP, Flores TR, Garcia LP, Chiavegatto Filho ADP, Thumé E, Facchini LA. Tendência temporal da falta de acesso aos serviços de saúde no Brasil, 19982013. Epidemiol Serv Saude 2016a; 25:777-787.

15. Brasil. Ministério da Saúde (MS). Portaria GM no 2.528, de 19 de outubro de 2006. Política Nacional de Saúde da Pessoa Idosa - PNSI. Diário Oficial da União; 2006.
16. Schmidt MI, Duncan BB, Hoffmann JF, Moura L, Malta DC, Carvalho RMSV. Prevalência de diabetes e hipertensão no Brasil baseada em inquérito de morbidade auto-referida, Brasil, 2006. Rev Saude Publica 2009; 43(Supl 2):74-82

17. Paskulin LMG, Valer DB, Vianna LAC. Utilização e acesso de idosos a serviços de atenção básica em Porto Alegre (RS, Brasil). Cien Saude Colet 2011; 16(6):2935-2944.

18. Facchini LA, Piccini RX, Tomasi E, Thumé E, Silveira DS, Siqueira FV, Rodrigues MA. Desempenho do PSF no Sul e no Nordeste do Brasil: avaliação institucional e epidemiológica da Atenção Básica à Saúde. Cien Saude Colet 2006; 11(3):669-681.

19. Rodrigues MAP, Facchini LA, Piccini RX, Tomasi E, Thumé E, Silveira DS, Paniz VMV, Siqueira FV. Uso de serviços ambulatoriais por idosos nas regiões Sul e Nordeste do Brasil. Cad Saude Publica 2008; 24(10):2267-2278

20. Dilélio AS, Tomasi E, Thumé E, Silveira DS, Siqueira FCV, Piccini RX, Silva SM, Nunes BP, Facchini LA. Lack of access and continuity of adult health care: a national population-based survey. Rev Saude Publica 2015; 49:1-15.

21. Dilélio AS, Tomasi E, Thumé E, Silveira DS, Siqueira FCV, Piccini RX, Silva SM, Nunes BP, Facchini LA. Padrões de utilização de atendimento médico-ambulatorial no Brasil entre usuários do Sistema Único de Saúde, da saúde suplementar e de serviços privados. Cad Saude Publica 2014; 30(12):2594-2606.

22. Nunes BP, Flores TR, Mielke, GI, Thumé E, Facchini LA. Multimorbidity and mortality in older adults: a systematic review and meta-analysis. Arch Gerontol Geriatr 2016; 67:130-138.

23. Lima-Costa MF. Epidemiologia do envelhecimento no Brasil. In: Rouquayrol MZ, Almeida Filho N, organizadores. Epidemiologia e saúde. Rio de Janeiro: MEDSI; 2003. p. 499-514.

24. Thumé E, Facchini LA, Wyshak G, Campbell P. The utilization of home care by the elderly in Brazil's primary health care system. Am J Public Health 2011; 101(5):868-874.

25. Brasil. Lei $n^{\circ} 8.842$, de 4 de janeiro de 1994. Dispõe sobre a política nacional do idoso, cria o Conselho Nacional do Idoso e dá outras providências. Diário Oficial da União 1994; 5 jan.

26. Fernández-Mayoralas G, Rodríguez V, Rojo F. Health services accessibility among Spanish elderly. Soc Sci Med 2000; 50(1):17-26.

27. Instituto Brasileiro de Defesa do Consumidor (IDEC). Nem vem que não tem [matéria na Internet]. Revista do IDEC; 2014 [acessado 2016 Ago 22]. Disponível em: http://www.idec.org.br/uploads/revistas_materias/pdfs/190-capa-planos-de-saude1.pdf

28. Brasil. Ministério da Saúde (MS). Agencia Nacional de Saúde Suplementar (ANS). Plano de cuidado para idosos na saúde suplementar. Rio de Janeiro: ANS; 2012.

29. Brasil. Decreto $n^{\circ} 7.508$, de 28 de junho de 2011. Regulamenta a Lei no 8.080 , de 19 de setembro de 1990 , para dispor sobre a organização do Sistema Único de Saúde - SUS, o planejamento da saúde, a assistência à saúde e a articulação interfederativa, e dá outras providências. Diário Oficial da União 2011; 29 jun. 
30. Almeida APSC, Lima LD. O público e o privado no processo de regionalização da saúde no Espírito Santo. Saude Debate 2015; 39(n. esp.):51-63.

31. Portela MC, Lima SML, Ugá MAD, Gerschman S, Vasconcellos MTL. Estrutura e qualidade assistencial dos prestadores de serviços hospitalares à saúde suplementar no Brasil. Cad Saude Publica 2010; 26(2):399408.

32. Brandão ALRBS, Giovanella L, Campos CEA. Avaliação da atenção básica pela perspectiva dos usuários: adaptação do instrumento EUROPEP para grandes centros urbanos brasileiros. Cien Saude Colet 2013; 18(1):103-114.

33. Lima-Costa MF, Loyola Filho AI. Fatores associados ao uso e à satisfação com os serviços de saúde entre usuários do Sistema Único de Saúde na Região Metropolitana de Belo Horizonte, estado de Minas Gerais, Brasil. Epidemiol Serv Saude 2008; 17(4):247-257.

34. Lima LD, Viana ALÁ, Machado CV, Albuquerque MV, Oliveira RG, Iozzi FL, Scatena JHG, Mello GA, Pereira AMM, Coelho APS. Regionalização e acesso à saúde nos estados brasileiros: condicionantes históricos e político-institucionais. Cien Saude Colet 2012; 17(11):2881-2892.

35. Almeida PF, Giovanella L, Mendonça MHM, Escorel S. Desafios à coordenação dos cuidados em saúde: estratégias de integração entre níveis assistenciais em grandes centros urbanos. Cad Saude Publica 2010; 26(2):286-298.

36. Oliver A, Mossialos E. Equity of access to health care: outlining the foundations for action. J Epidemiol Community Health 2004; 58(8):655-658.

Artigo apresentado em 18/03/2018

Aprovado em 27/11/2018

Versão final apresentada em 29/11/2018 